REVISTA DE LA ESCUELA DE CIENCIAS DE LA EDUCACIÓN, AÑO 16, NRO. 15, VOL. 2, JULIO A DICIEMRE DE 2020. PÁGINAS 34-44. ISSN 2362-3349 (EN LÍNEA). HÁBITOS DE VIDA SALUDABLES Y RENDIMIENTO ESCOLAR EN ESTUDIANTES UNIVERSITARIOS. MARIO MIGUEL OJEDA RAMÍREZ. JOSÉ JUAN MUÑOZ LEÓN. ERNESTO PEDRO MENÉNDEZ ACUÑA

\title{
HÁBITOS DE VIDA SALUDABLES Y RENDIMIENTO ESCOLAR EN ESTUDIANTES UNIVERSITARIOS
}

\author{
Mario Miguel Ojeda Ramírez* \\ Facultad de Estadística e Informática de la Universidad Veracruzana, México \\ mojeda@uv.mx \\ José Juan Muñoz León ** \\ Facultad de Estadística e Informática de la Universidad Veracruzana, México \\ juanmunoz@uv.mx \\ Ernesto Pedro Menéndez Acuña *** \\ Facultad de Matemáticas y Facultad de Estadística e Informática de la Universidad Veracruzana, México \\ emenendez@uv.mx
}

Recibido: 18/09/2019 Aceptado: 23/12/2019

\section{Resumen}

Es incuestionable que los buenos comportamientos respecto a la salud tienen beneficios, algunos muy tangibles y otros no tan claros. Los hábitos de vida saludables (HVS) se deben adquirir en el marco de la familia, pero se deben reforzar y promover en el ámbito escolar. Generalmente esto se realiza en la educación primaria y secundaria. No obstante, se tiene evidencia que en la educación superior los HVS no se practican de la mejor forma. Se ha señalado que esto último puede impactar en el rendimiento académico. En este estudio se presenta la modelación del rendimiento escolar dependiente de los hábitos de vida saludables (HVS) --alimentación (A), actividad física y ejercicio (AE), manejo del estrés (ME), apoyo interpersonal (AI), autorrealización (AU) y responsabilidad en salud (RS)- considerando la influencia del sexo y el nivel de avance en los estudios. Para la medición de los HVS se utilizó una adaptación de la prueba de Walker, Sechrist \& Pender (1987). Se obtuvo una muestra por cuotas de estudiantes de las carreras de Estadística, Economía, Geografía, Redes y Servicios de Computo, Ingeniería de Software, y Tecnologías Computacionales, garantizando representatividad equivalente de sexo ( $\mathrm{M}=$ masculino y $\mathrm{F}=$ femenino) y nivel de avance en el programa educativo ( $\mathrm{l}=$ inicial y

*Doctor en Ciencias Matemáticas por la Universidad de la Habana, Maestro en Ciencias con mención en Estadística por el Colegio de Posgraduados y Licenciado en Estadística por la Universidad Veracruzana. Profesor de esta última institución desde 1981. Es miembro regular de la Academia Mexicana de Ciencias, Investigador Nacional Nivel I y profesor de perfil deseable reconocido por el PROMEP hasta el 2020. Actualmente, es profesor titular en la Facultad de Estadística e Informática de la Universidad Veracruzana en México.

**Doctor en Didáctica de las Matemáticas por la por la Universitat Autónoma de Barcelona, Maestro en Didáctica de las Matemáticas por la Universitat Autónoma de Barcelona, Maestro en Gestión de la Calidad por la Universidad Veracruzana y Licenciado en Matemáticas (2003) por la Universidad Veracruzana. Actualmente es profesor titular en la Facultad de Estadística e Informática de la Universidad Veracruzana en México.

***Doctor en Ciencias Matemáticas por la Universidad de La Habana y Licenciado en Matemática por la Universidad de La Habana. Actualmente es profesor en la Facultad de Matemáticas y en la Facultad de Estadística e Informática de la Universidad Veracruzana en México. 
REVISTA DE LA ESCUELA DE CIENCIAS DE LA EDUCACIÓN, AÑO 16, NRO. 15, VOL. 2, JULIO A DICIEMRE DE 2020. PÁGINAS 34-44. ISSN 2362-3349 (EN LÍNEA). HÁBITOS DE VIDA SALUDABLES Y RENDIMIENTO ESCOLAR EN ESTUDIANTES UNIVERSITARIOS. MARIO MIGUEL OJEDA RAMíREZ. JOSÉ JUAN MUÑOZ LEÓN. ERNESTO PEDRO MENÉNDEZ ACUÑA

A=avanzado). Los datos de rendimiento escolar se obtuvieron del registro de calificaciones; se tomó el promedio general. Se compararon los HVS no encontrándose diferencias entre programas educativos. Se construyó e interpretó un índice de HVS a través de la técnica de componentes principales y se postuló y ajustó un modelo de regresión simple para cada grupo en la interacción de sexo y nivel de avance. Los resultados muestran que el rendimiento escolar tiene una dependencia de los HVS al considerar una agrupación de los estudiantes por sexo y nivel de avance en el programa educativo.

Palabras clave: Actividad física - Educación superior - Hábitos alimentarios - Modelación de regresión - Rendimiento académico.

\begin{abstract}
:
It is unquestionable that good behaviors regarding health have benefits, some very tangible and others not so clear. Healthy living habits (HVS) must be acquired within the family framework, but they must be reinforced and promoted in the school setting. Generally, this is done in primary and secondary education. However, there is evidence that in higher education HVS are not practiced in the best way. It has been pointed out that the latter can impact on academic performance. This study presents the modeling of school performance dependent on healthy lifestyle habits (HVS) -feeding (A), physical activity and exercise (AE), stress management (ME), interpersonal support (AI), self-realization (AU) and responsibility in health (RS)- considering the influence of sex and the level of advance in the studies. An adaptation of the Walker, Sechrist \& Pender test (1987) was used to measure the HVS. A sample was obtained by student fees from the careers of Statistics, Economics, Geography, Networks and Services of Computation, Software Engineering, and Computational Technologies, guaranteeing equivalent representation of $\operatorname{sex}(\mathrm{M}=$ male and $\mathrm{F}=$ female) and level of advancement in the educational program (I $=$ initial and $\mathrm{A}=$ advanced). The school performance data was obtained from the record of grades; the general average was taken. The HVS were compared with no differences between educational programs. An HVS index was constructed and interpreted through the principal component technique and a simple regression model was postulated and adjusted for each group in the interaction of sex and advancement level. The results show that school performance is dependent on HVS when considering a grouping of students by sex and level of advancement in the educational program.
\end{abstract}

Keywords: Academic performance - Dietary habits - Higher education - Physical activity - Regression modeling.

\title{
Introducción
}

El conjunto de pautas y hábitos de comportamiento cotidianos de una persona, que son patrones de conducta individuales -los cuales pueden constituirse en factores de seguridad o riesgo- se conocen como hábitos de vida. La práctica de estos hábitos regularmente es determinante en muchos ámbitos de desempeño. Por tal motivo, desde la promoción de una vida saludable, se ha optado por enfatizar en los llamados hábitos de vida saludables (HVS), que incluyen conductas de salud, patrones de conducta, creencias, conocimientos y acciones para mantener, restablecer o mejorar la salud y por ende la calidad de vida. Los HVS son producto de dimensiones personales, ambientales y sociales, que emergen del sujeto mismo en su tránsito por la vida. Los principales espacios donde se promueven los HVS son la familia y la escuela. La familia es definitoria de los HVS y la escuela coadyuva y da soporte al desarrollo de los HVS. En la educación se promueven los HVS hasta la educación media, incluso con temáticas y programas que forman parte del currículo, pero se entiende que al llegar al nivel universitario los individuos están plenamente formados y son responsables de no correr riesgos y darle soporte permanente a los HVS. Sin embargo, como lo señalan Díaz Cárdenas, Martínez Redondo \& Zapata Teherán, (2017), este contexto educativo puede representar un riesgo para la salud:

Tal y como se evidencian cuadros depresivos en universitarios en comparación con la población general, aumento en la tasa de intentos de suicidio y suicidio consumado en jóvenes, cambios en hábitos alimentarios que generan trastornos en el metabolismo, y problemas de salud como gastritis, cefaleas y hasta ansiedad, por algunas causas, como el carácter estresor de la carrera escogida, dificultades para adaptarse a hábitos de estudio universitarios y conciliar estudios con otras demandas como compromisos familiares (Díaz Cárdenas, et. al., 2007, p.140). 
REVISTA DE LA ESCUELA DE CIENCIAS DE LA EDUCACIÓN, AÑO 16, NRO. 15, VOL. 2, JULIO A DICIEMRE DE 2020. PÁGINAS 34-44. ISSN 2362-3349 (EN LÍNEA). HÁBITOS DE VIDA SALUDABLES Y RENDIMIENTO ESCOLAR EN ESTUDIANTES UNIVERSITARIOS. MARIO MIGUEL OJEDA RAMÍREZ. JOSÉ JUAN MUÑOZ LEÓN. ERNESTO PEDRO MENÉNDEZ ACUÑA

Esto nos hace enfatizar que la vida de los estudiantes universitarios se ve afectada por las dinámicas escolares que establecen horarios de dedicación y niveles de exigencia, los cuales hacen que el mantenimiento de los HVS se constituya en un reto adicional. Los citados autores también señalan que: "estos eventos pueden comprometer el rendimiento académico y a largo plazo su calidad de vida relacionada con la salud" (Díaz Cárdenas, et. al., 2007, p. 140).

Gallardo-Escudero, et al. (2015) señala que la etapa de los estudios superiores implica cambios de diversa índole: emocionales y fisiológicos, así como aquellos derivados de un nuevo ambiente y el ingreso a una vida de mayor independencia de la familia, lo que van a determinar comportamientos y costumbres, que se pueden convertir en hábitos de riesgo, muchos de los cuales, al mantenerse a lo largo de la vida, repercutirán en la salud. Con esta motivación realizaron un estudio del estilo de vida, considerando el consumo de alcohol, tabaco y prácticas de actividad física; los sujetos fueron 55 mujeres estudiantes, de 18 a 31 años, segmentadas en dos grupos, de la Universidad de Granada, en España. Se aplicó un cuestionario de estilo de vida evaluando el tipo y frecuencia de consumo de alcohol, cantidad de cigarrillos consumidos diariamente y niveles de actividad física, considerando las categorías: sedentaria, ligera, moderada e intensa. Los resultados mostraron que el consumo de alcohol es mayor en el grupo de mayor edad, y con preferencia beben cerveza y vino; el grupo de las más jóvenes presentó un patrón de consumo centrado en los fines de semana, siendo las bebidas destiladas las consumidas preferentemente. Una tercera parte de la población fuma con un incremento en el número de cigarros conforme aumenta la edad. Encontraron una asociación entre tabaco y alcohol; es decir, a mayor consumo de uno mayor consumo del otro; asimismo destacan que 9 de cada 10 del grupo de menor edad y 5 de cada 10 del grupo de mayor edad tienen una actividad física que va de sedentaria a ligera. A partir de estos resultados derivaron la recomendación de crear mayor conciencia en esta población sobre los beneficios del abandono del consumo del alcohol y del tabaco y de la práctica regular de ejercicio físico, por lo que sugieren desarrollar protocolos de intervención educativa que promuevan los HVS en el ámbito universitario.

En este mismo sentido, Rodríguez, Restrepo y Deossa-Restrepo (2015) realizaron un estudio de estilos de vida saludable en jóvenes, señalando el interés que este conocimiento tiene para prevenir la aparición de enfermedades crónicas no trasmisibles; concretamente evaluaron los conocimientos y prácticas sobre alimentación, salud y ejercicio de estudiantes universitarios y la relación de factores como sexo y nivel socioeconómico; fue un estudio de muestreo descriptivo de carácter exploratorio, transversal, con una muestra de 210 hombres y 210 mujeres, estudiantes de algunas universidades de la ciudad de Medellín. Utilizaron postestratificación del nivel socioeconómico. A través de análisis estadísticos multivariantes detectaron diferencias de conocimientos y aspectos relacionados al control de peso altamente significativas entre sexos; asimismo se detectó diferencia significativa entre sexos, en lo referente a las creencias sobre hábitos alimentarios y de salud. A partir de un análisis de correspondencia múltiple encontraron asociación entre el conocimiento sobre el índice de masa corporal (IMC) y el conocimiento sobre sus implicaciones en la salud; igualmente, entre el estrato socioeconómico y el conocimiento relacionado con el IMC. Señalaron, concluyendo, que los estudiantes universitarios tienen una percepción regular sobre sus conocimientos acerca de hábitos de alimentación saludable, actividad física y control de peso, así como sus implicaciones en salud; es menor el conocimiento entre hombres y en el estrato socioeconómico bajo.

Es por esta razón que, en el contexto de la educación superior, medir, monitorear y estudiar los HVS, se ha convertido en un tema relevante (Gillis, 1997; Guerrero, et. al, 2014). Se reporta que la alimentación de los universitarios no presenta hábitos adecuados: comidas rápidas y dietas con altos contenidos calóricos; el ejercicio físico es rara vez reportado en sus prácticas cotidianas. En este marco, las mujeres son las que mejores prácticas saludables declaran (Lema, et. al, 2009), aunque Cutillas et. al (2013) no encontraron diferencias significativas al considerar la variable sexo. Se puede concluir que los universitarios tienen conciencia sobre la importancia de los hábitos de vida saludables, aunque sus hábitos sean contradictorios (Sánchez-Ojeda y De Luna-Bertos, 2015).

Existen evidencias de que los HVS están directamente relacionados con el nivel de aprovechamiento o rendimiento escolar. Deliens, Clarys, De Bourdeaudhuij \& Deforche (2013) estudiaron a 101 estudiantes universitarios belgas de primer año. Identificaron el peso y el comportamiento en salud relacionado con el rendimiento académico. Aplicaron un cuestionario de comportamiento de salud en línea. Como medida del rendimiento académico tomaron el promedio general de calificaciones al fin de año. A través de análisis multivariantes encontraron que ser de sexo masculino, menor en edad, con aumento de peso, índice de masa corporal y circunferencia de la cintura alto, más distracciones entre semana, estar a dieta, comer con mayor frecuencia en el restaurante estudiantil, con un mayor consumo de refrescos y papas fritas, y una mayor frecuencia de consumo de alcohol, predijeron un menor promedio de calificaciones, con lo que se mostró que el rendimiento académico está asociado a una amplia gama de comportamientos relacionados con el peso y la salud; señalan que los estudios futuros deberían investigar si las intervenciones que promueven comportamientos saludables entre los estudiantes también podrían tener un impacto positivo en el desempeño académico. 
REVISTA DE LA ESCUELA DE CIENCIAS DE LA EDUCACIÓN, AÑo 16, NRO. 15, VOL. 2, JULIO A DICIEMRE DE 2020. PÁGINAS 34-44. ISSN 2362-3349 (EN LÍNEA). HÁBITOS DE VIDA SALUDABLES Y RENDIMIENTO ESCOLAR EN ESTUDIANTES UNIVERSITARIOS. MARIO MIGUEL OJEDA RAMíREZ. JOSÉ JUAN MUÑOZ LEÓN. ERNESTO PEDRO MENÉNDEZ ACUÑA

Sabemos que la actividad física mejora la circulación sanguínea general, aumenta el flujo de sangre al cerebro y aumenta los niveles de norepinefrina y endorfinas, todas las cuales pueden reducir el estrés, mejorar el estado de ánimo, inducir un efecto calmante después del ejercicio y, tal vez, mejorar el rendimiento académico. La actividad física en las escuelas también proporciona beneficios sociales, promueve el respeto a las reglas, la disciplina, persistencia y la necesidad de la práctica, aspectos que podrían resultar a su vez en beneficios académicos. Por esta razón, es probable que la actividad física se perciba más relacionada con el rendimiento escolar de lo que en realidad lo está. Los maestros, padres y gestores educativos coincidirán con la suposición de que la actividad física es razonable que ayude a los escolares a tener un mejor desempeño en la escuela. Además, se sabe que los adolescentes físicamente activos tienen menos probabilidades de intentar suicidarse, adoptar conductas riesgosas, etc., todo lo cual puede estar asociado a mejores resultados académicos. Es así que, revisar el estado de la investigación sobre la asociación entre la actividad física y los resultados académicos tiene una fuerte tradición. Taras (2005) revisó 14 estudios publicados durante una década previa a su investigación: demostró que puede haber algunas mejoras a corto plazo (como en la concentración), pero esas mejoras no se mantienen a largo plazo y no impactan significativamente en los logros académicos. Concluye que el efecto de la actividad física sobre el rendimiento escolar no está bien fundamentado y requiere más elucidación.

Trockel, Barnes y Egget (2000) analizaron el efecto de variables relacionadas con la salud sobre los promedios de calificaciones de una muestra aleatoria de 200 estudiantes que viven en residencias universitarias en una universidad privada. El conjunto de variables incluyó hábitos de ejercicio, alimentación y sueño; estados de ánimo; estrés percibido; gestión del tiempo; apoyo social; hábitos espirituales o religiosos y número de horas trabajadas por semana; también incluyeron las variables de sexo y edad. De las variables consideradas, los hábitos de sueño, particularmente la hora de levantarse, tuvieron el mayor efecto sobre los promedios de calificaciones. Los estudiantes que se levantaban más tarde tuvieron una tendencia hacia calificaciones promedio más bajas. Las variables asociadas con los promedios de calificaciones de los estudiantes de primer año fueron el entrenamiento y el estudio de material sobre formación espiritual. El número de horas trabajadas, para obtener ingresos o como actividades voluntarias, por semana, se asoció con calificaciones promedio más bajas. En otro estudio de este tipo, con el propósito de asociar rendimiento académico y la calidad de vida relacionada con la salud, Díaz Cárdenas et al. (2017) realizaron una investigación con 347 estudiantes de odontología de la Universidad de Cartagena. Se diseñó y aplicó encuesta autoadministrada considerando variables sociodemográficas y de calidad de vida relacionada con la salud. El rendimiento académico fue obtenido a través de registros oficiales de la institución. Se estimaron prevalencias y asociaciones entre variables, encontrándose que un tercio, aproximadamente, de los estudiantes presentaron bajo rendimiento académico, y los principales motivos de pérdidas de asignaturas fueron poco tiempo dedicado a estudiar (2 de cada 10) y metodología académica utilizada (1 de cada 10). Según el cuestionario de calidad de vida relacionada con la salud, 4 de cada 10 percibió que presenta una buena calidad y más de la mitad declaró sentirse bastante bien con su salud. Se encontró que la asociación entre los estudiantes con bajo rendimiento académico y compromiso del dominio social fue significativa, por lo que concluyen que se "debe mirar de una manera holística el desarrollo académico del estudiante en aras del mejoramiento de su calidad de vida” (Díaz Cárdenas et al., 2017, p. 139).

Se ha aseverado que la práctica de un deporte o actividad física de forma regular está estrechamente relacionada con un buen rendimiento académico (Shariati y Bakhtiari, 2011) aunque hay quienes no han encontrado relación alguna entre la práctica deportiva y el rendimiento académico (Yu, Chan, Cheng, Sung y Hau, 2006). A este respecto Capdevila Seder, Bellmunt Villalonga y Hernando Domingo (2015) señalan que los sujetos deportistas tienen mejor rendimiento académico, mejores hábitos de estudio y dedican menos tiempo al ocio sedentario; además, dentro de los hábitos de estudio, los sujetos deportistas planifican mejor su tiempo libre y presentan mejor actitud frente a los estudios. Recientemente se ha reportado que el estilo de vida está fuertemente asociado con el rendimiento académico de los estudiantes en el nivel básico. Esto lleva a que la promoción del estilo de vida saludable podría mejorar tanto la salud como los resultados educativos. Con esto se ha concluido que iniciativas de promoción de la salud dirigidas al estilo de vida pueden tener un efecto mayor en el rendimiento académico. También se ha encontrado un efecto significativo del ejercicio en dos dominios de rendimiento académico, a saber, mejor concentración en las clases y puntualidad en la asistencia a las clases, pero sin efecto significativo en el rendimiento global (Faught et. al, 2017; Alsabih et. al, 2018).

Eventualmente la relación entre HVS puede estar mediada por el sexo y por el nivel de avance en e programa de estudios, ya que se ha dicho que a una mayor formación se tiene una mayor conciencia respecto a la importancia de los HVS. En este marco, el principal indicador objetivo del rendimiento escolar es el promedio de calificaciones obtenido en un periodo dado. Se ha explorado alguna relación entre la HVS y el rendimiento escolar, pero esto no se ha llevado hasta el nivel de postular un modelo y evaluar la consistencia de las hipótesis señaladas. Por estos motivos, en este estudio se presenta la modelación de los HVS (alimentación (A), actividad física y ejercicio (AE), manejo del estrés (ME), apoyo interpersonal 
REVISTA DE LA ESCUELA DE CIENCIAS DE LA EDUCACIÓN, AÑO 16, NRO. 15, VOL. 2, JULIO A DICIEMRE DE 2020. PÁGINAS 34-44. ISSN 2362-3349 (EN LÍNEA). HÁBITOS DE VIDA SALUDABLES Y RENDIMIENTO ESCOLAR EN ESTUDIANTES UNIVERSITARIOS. MARIO MIGUEL OJEDA RAMÍREZ. JOSÉ JUAN MUÑOZ LEÓN. ERNESTO PEDRO MENÉNDEZ ACUÑA

$(A I)$, autorrealización $(A U)$ y responsabilidad en salud $(R S)$ ) de estudiantes universitarios con el rendimiento escolar considerando la eventual influencia del sexo y el nivel de avance en los estudios.

\section{Contexto del estudio y justificación}

La Universidad Veracruzana (UV) es una de las 5 principales IES estatales de México, por su tamaño, número de programas y dispersión geográfica. En el estado de Veracruz es la principal institución de educación y cuenta con una matrícula de aproximadamente 63 mil estudiantes, que representa un poco más del $30 \%$ de los estudiantes de educación superior de esta entidad federativa mexicana. En la región Xalapa se ubican la mayoría de los programas educativos, estudiantes y capacidad de investigación, representando aproximadamente el $50 \%$ de la matrícula total. Es una IES que pertenece a las universidades públicas estatales (UPES) de la república mexicana. Cuenta con 166 programas de licenciatura 130 programas de posgrado distribuidos en las áreas académicas de humanidades, ciencias de la salud, técnica, artes, biológico-agropecuarias y económico administrativa. La unidad académica de economía y estadística, ubicada en la región Xalapa, perteneciente al área económico administrativa, se integra por dos facultades: la Facultad de Economía, que ofrece dos programas educativos (Economía y Geografía) y la Facultad de Estadística e Informática, con cuatro programas educativos (Estadística, Redes y Servicios de Computo, Ingeniería de Software, y Tecnologías Computacionales). La población estudiantil de esta unidad académica es de aproximadamente 2000 estudiantes. Por la actividad académica de los investigadores y por diversos estudios no publicados se conoce de la necesidad de investigar a mayor profundidad sobre los HVS y su impacto en el rendimiento académico. La primera pregunta es si existen diferencias entre las carreras que se ofrecen en esta unidad académica respecto a los HVS. Otra motivación es conocer si hay diferencias en los promedios de calificaciones entre carreras. Se quiere, también, estudiar las diferencias entre estas variables segmentando la muestra tanto por nivel de estudios como por la variable sexo. Y finalmente saber sobre la relación entre HVS y rendimiento escolar: conocer si esta relación es la misma dependiendo del nivel de estudios y el sexo. Los resultados de esta investigación podrán servir a las autoridades educativas y a quienes dirigen los programas de atención integral de los estudiantes universitarios, al mismo tiempo que se dará más de evidencia sobre el impacto de los HVS sobre el rendimiento académico en la educación superior.

\section{Metodología}

Se obtuvo una muestra por cuotas de 108 estudiantes de las carreras de Estadística (17), Economía (17), Geografía (20), Redes y Servicios de Computo (17), Ingeniería de Software (18), Tecnologías Computacionales (19), garantizando representatividad equivalente de sexo (M=masculino (60) y $F=$ femenino (48)) y nivel de avance en el programa educativo (I=inicial ( 59 ) y $A=a v a n z a d o ~(49)$ ). Para la medición de los hábitos saludables se adaptó el test de Walker, Sechrist \& Pender (1987), el cual es ampliamente conocido (Gillis, 1997). Los datos de rendimiento escolar se obtuvieron del registro de calificaciones; se tomó el promedio general. Se obtuvieron los puntajes de alimentación (A), actividad física y ejercicio $(A E)$, manejo del estrés (ME), apoyo interpersonal $(A I)$, autorrealización $(A U)$ y responsabilidad en salud (RS), así como un puntaje total (TOT), los cuales se compararon de manera univariante, no encontrándose diferencias entre programas educativos. Para estudiar la asociación entre los puntajes HVS; es decir A, AE, ME, Al, AU y RS, se realizó un análisis de componentes principales. Se interpretó un índice de HVS y se postuló y ajustó un modelo de regresión, tomando como variable explicatoria el puntaje TOT, considerando cuatro grupos: G1=femenino-inicial (n1=27), G2=femenino-avanzado (n2=21), G3=masculinoinicial (n3=32) y G4=masculino-avanzado (n4=28). Se interpretaron las rectas de regresión ajustadas.

\section{Resultados}

En la Figura 1 se despliegan las distribuciones de las dos variables más importantes en el estudio, donde vemos que la variable TOT en esta muestra sigue una distribución aproximadamente normal con media 66.06 y desviación estándar 9.61; la variable del promedio de calificaciones tiene una distribución que es menos evidente que sea normal con una mayor frecuencia de promedios altos; la media de esta última variable resultó ser 7.90 con una desviación estándar de 1.22. 
REVISTA DE LA ESCUELA DE CIENCIAS DE LA EDUCACIÓN, AÑO 16, NRO. 15, VOL. 2, JULIO A DICIEMRE DE 2020. PÁGINAS 34-44. ISSN 2362-3349 (EN LÍNEA). HÁBITOS DE VIDA SALUDABLES Y RENDIMIENTO ESCOLAR EN ESTUDIANTES UNIVERSITARIOS. MARIO MIGUEL OJEDA RAMÍREZ. JOSÉ JUAN MUÑ̃OZ

\section{Histogra de puntaje TOT}

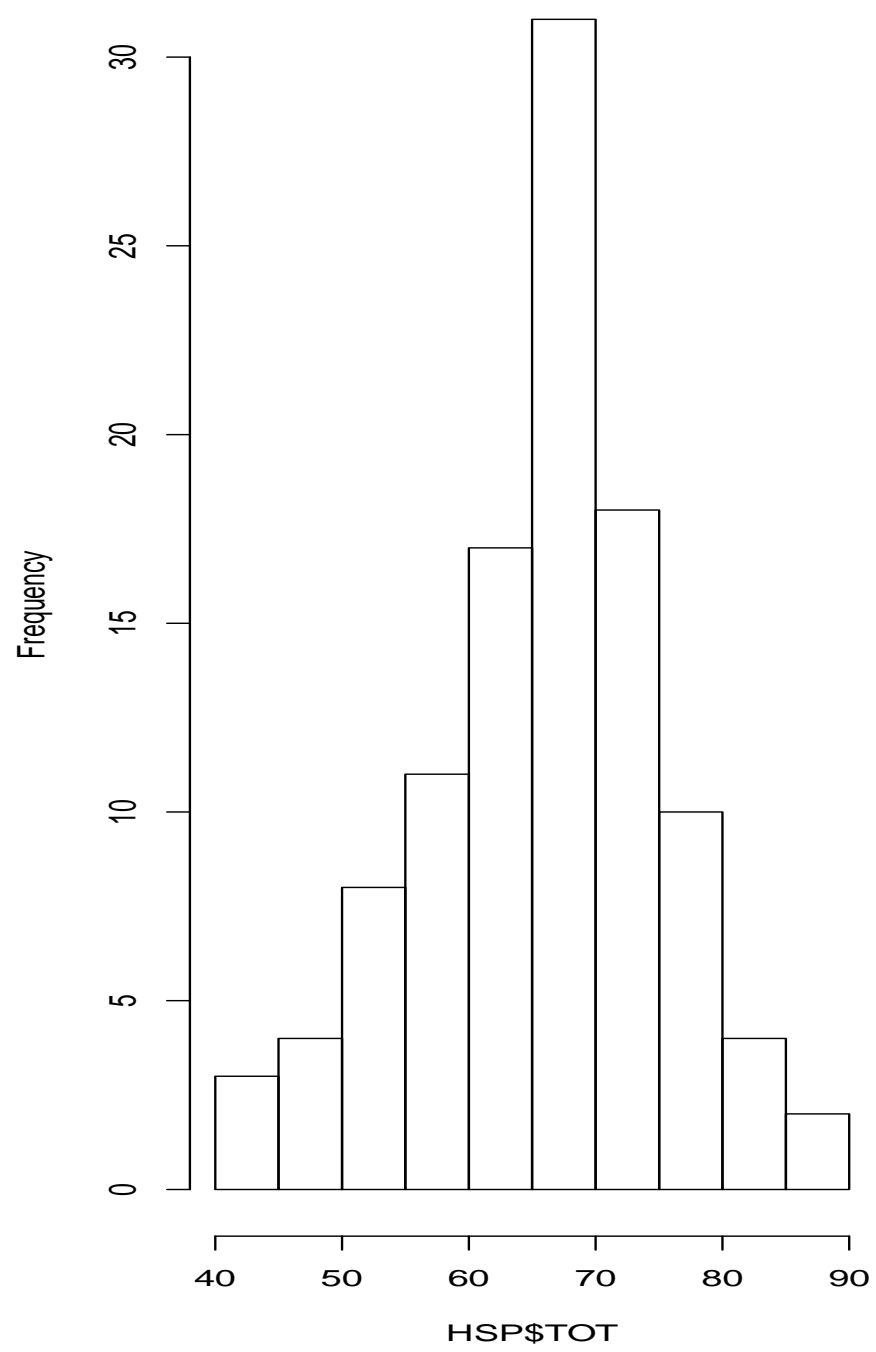

Histogra del promedio académico

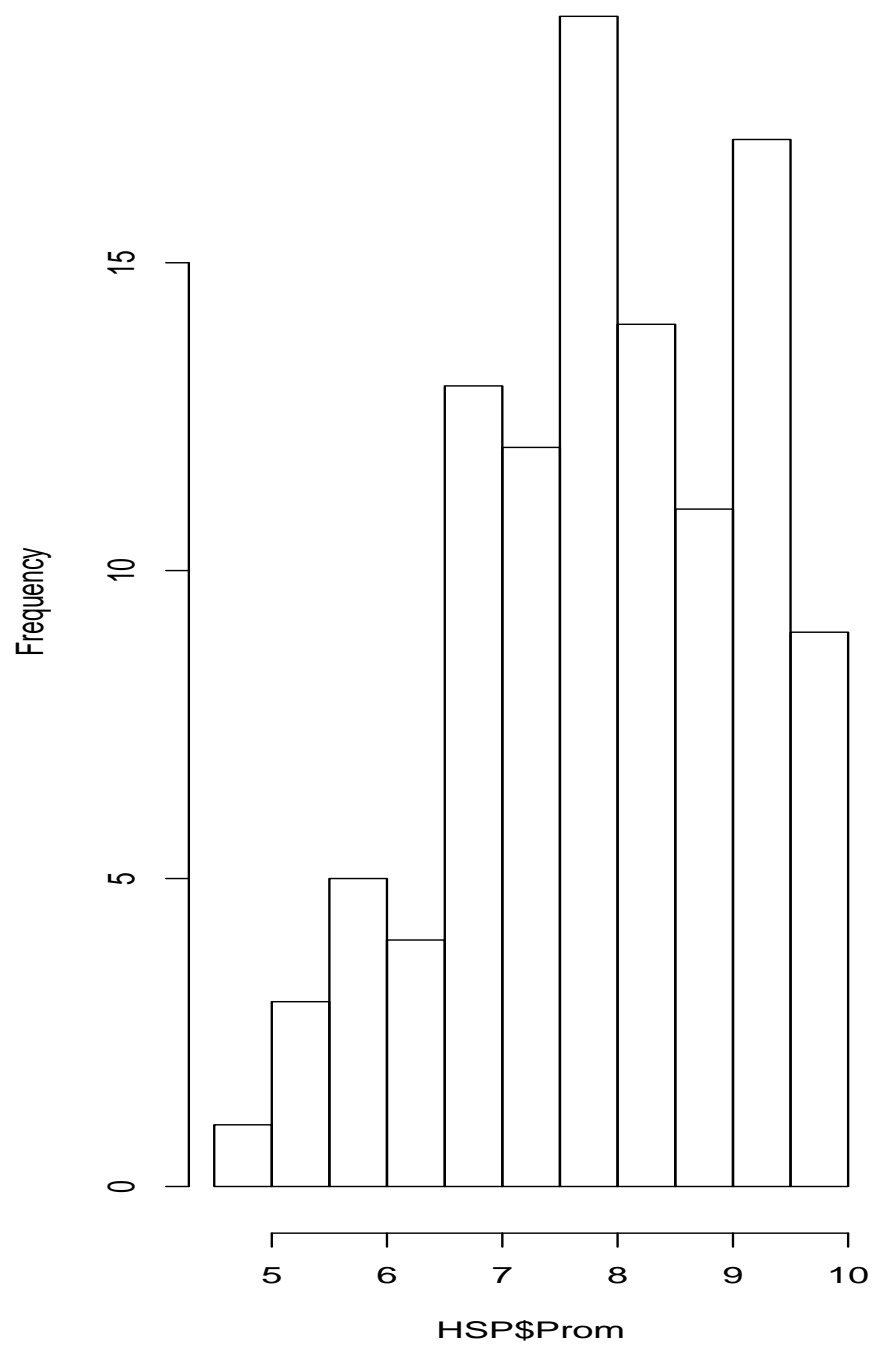

Figura 1. Distribución del puntaje TOT de HVS y el promedio general de los estudiantes encuestados.

Se realizó la comparación de estos puntajes en los grupos formados realizando un análisis de la varianza no obteniéndose diferencias significativas en ninguno de los casos. Esto se puede constatar en la Figura 2. Para el primer caso la F calculada resultó 0.427 con un valor de probabilidad empírica $p=0.734$, y para el segundo caso resultó 1.045 con el correspondiente $p=0.376$. Se realizaron la validación de los supuestos de normalidad y homogeneidad de varianzas no encontrándose evidencia de violación alguna. 
REVISTA DE LA ESCUELA DE CIENCIAS DE LA EDUCACIÓN, AÑO 16, NRO. 15, VOL. 2, JULIO A DICIEMRE DE 2020. PÁGINAS 34-44. ISSN 2362-3349 (EN LÍNEA). HÁBITOS DE VIDA SALUDABLES Y RENDIMIENTO ESCOLAR EN ESTUDIANTES UNIVERSITARIOS. MARIO MIGUEL OJEDA RAMÍREZ. JOSÉ JUAN MUÑOZ

\section{Comparativo del puntaje TOT}

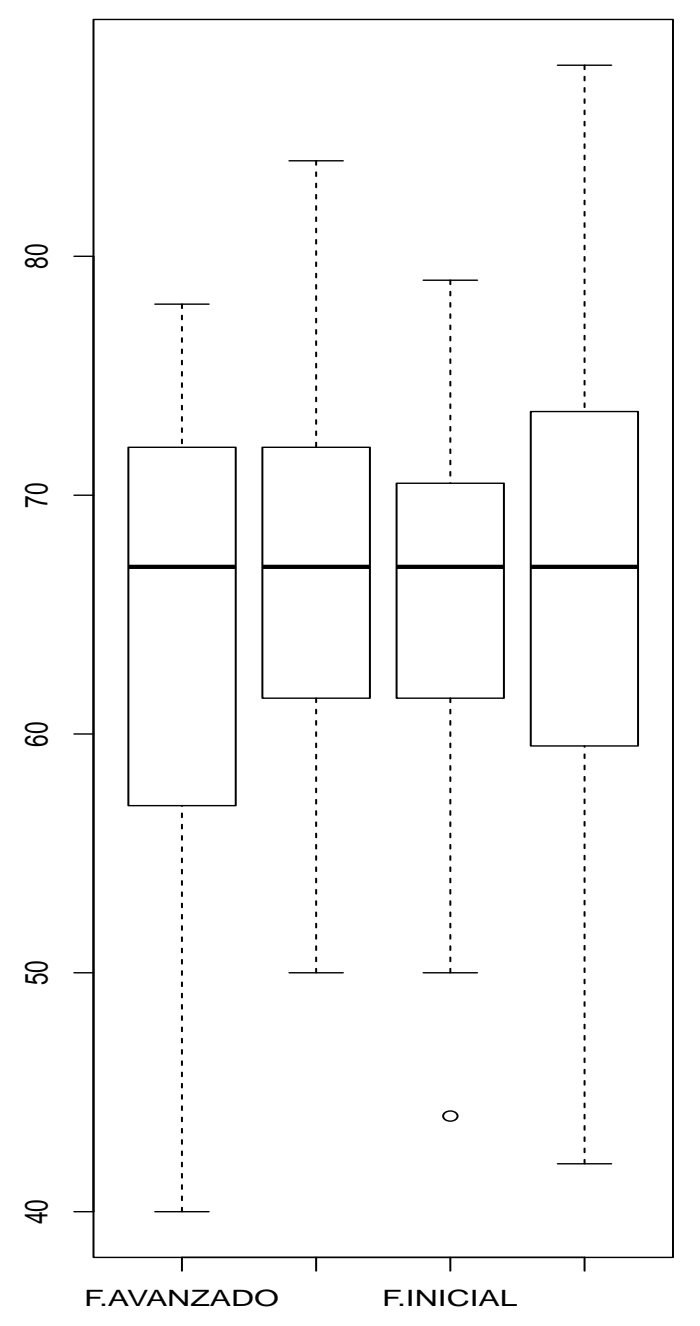

Comparativo de los promedios

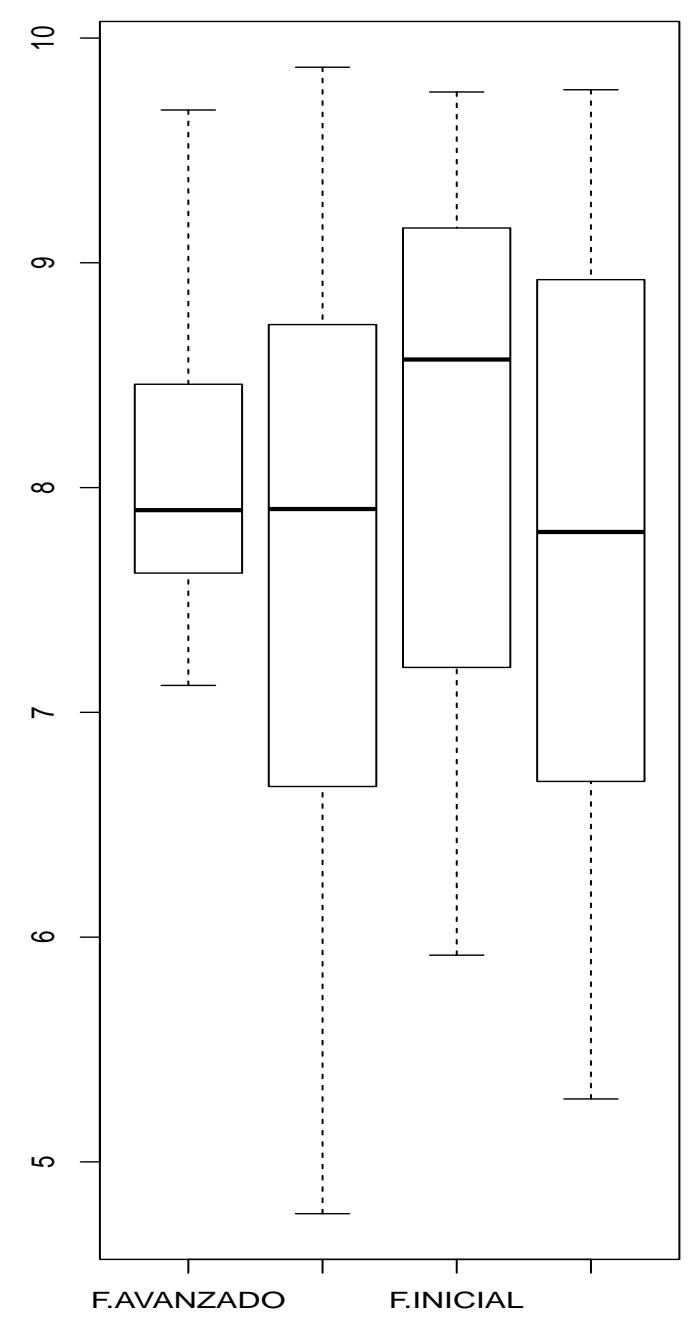

Figura 2. Gráficos de caja comparativos de los puntajes TOT de HVS y el promedio de calificaciones de los estudiantes en la muestra.

Cuando estudiamos la asociación de estas dos variables sobre toda la muestra encontramos un coeficiente de correlación de Pearson de 0.12, lo que indica que globalmente no existe una correlación importante. No obstante, al segmentar el colectivo en los cuatro grupos (G1, G2, G3 y G4) encontramos los siguientes coeficientes de correlación: $0.12,0.08,0.50$ y -0.37 , lo cual nos indica un efecto conjunto de las variables sexo y nivel de avance en los estudios, lo que produce un comportamiento diferenciado. Al realizar el estudio de la correlación entre los puntajes HVS; es decir A, AE, ME, AI, AU y RS, considerando el promedio de calificaciones, obtenemos que hay pocas correlaciones importantes. 
REVISTA DE LA ESCUELA DE CIENCIAS DE LA EDUCACIÓN, AÑO 16, NRO. 15, VOL. 2, JULIO A DICIEMRE DE 2020. PÁGINAS 34-44. ISSN 2362-3349 (EN LÍNEA). HÁBITOS DE VIDA SALUDABLES Y RENDIMIENTO ESCOLAR EN ESTUDIANTES UNIVERSITARIOS. MARIO MIGUEL OJEDA RAMÍREZ. JOSÉ JUAN MUÑOZ LEÓN. ERNESTO PEDRO MENÉNDEZ ACUÑA

La Figura 3 muestra el diagrama matricial de las asociaciones donde se destaca que hay pocas correlaciones mayores de 0.40 , que son las ubicadas en el segmento de $\mathrm{ME}, \mathrm{Al}, \mathrm{AUR}$ y RS, que son $0.4039,0.4813,0.5062$ y 0.54 , respectivamente.
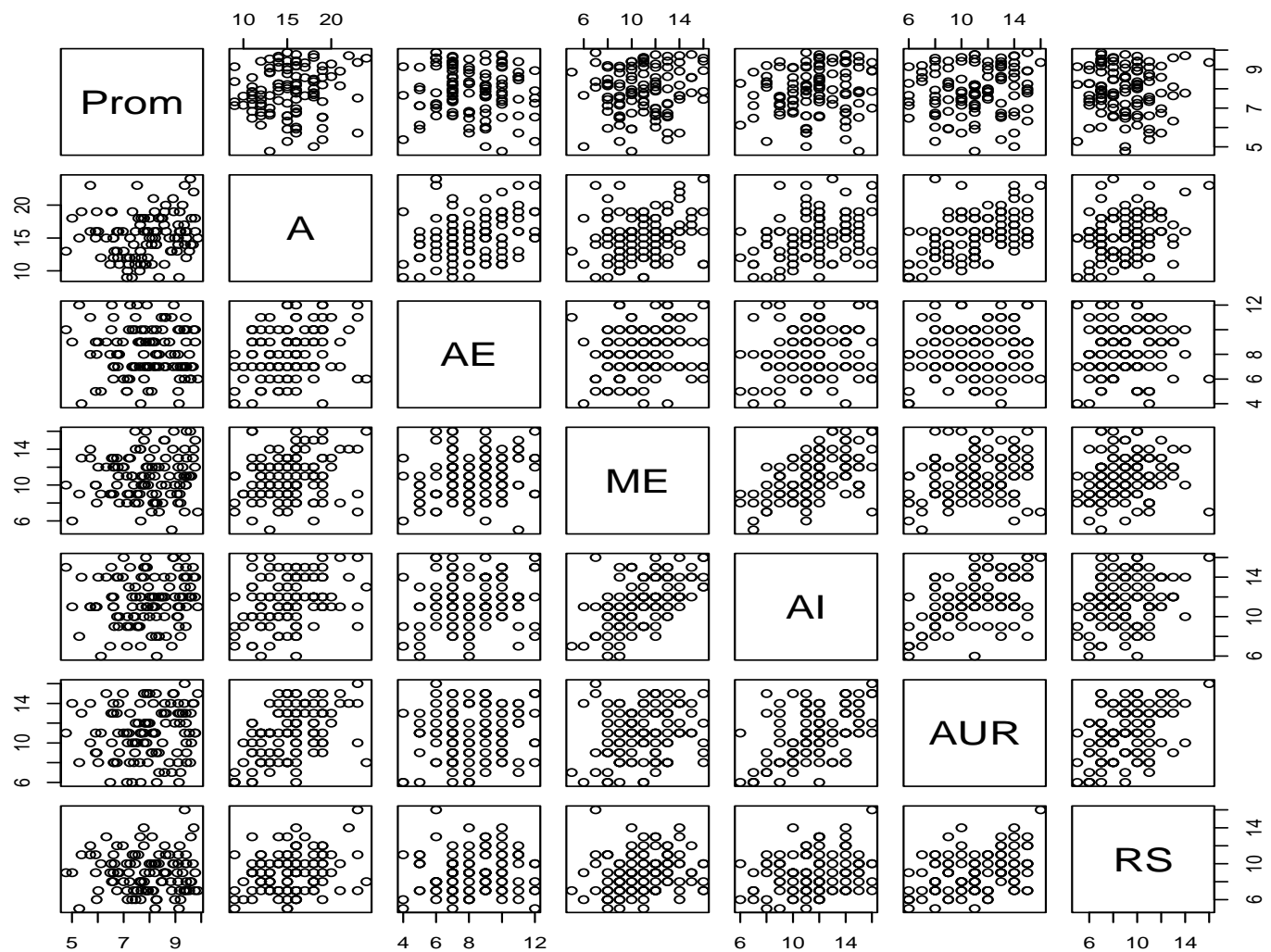

Figura 3. Diagrama matricial de los diferentes puntajes de HVS y el promedio de calificaciones de los estudiantes en la muestra.

El análisis de componentes principales nos arrojó que con dos componentes se explicó el $58 \%$ de la varianza total; el primer componente CP1 fue claramente un componente de tamaño, correlacionado de manera casi perfecta con el puntaje TOT; este componente explicó el $41 \%$ de la varianza multivariante total; el segundo componente reflejó la existencia de opiniones contradictorias en la muestra: se corresponde con estudiantes que tienen bajos puntajes de actividad y ejercicio ( $A E)$, y manejo del estrés (ME), pero altos puntajes de autorrealización (AU) y responsabilidad en salud (RS), y viceversa. A continuación, en la Figura 4 , se muestra un diagrama matricial de las distribuciones y asociaciones considerando la segmentación de los grupos formados por las variables sexo y nivel de avance en los estudios, considerando el promedio el componente principal 1 y el puntaje total de HVS. Es notorio que TOT y CP1 están altamente correlacionados, por lo que se decidió estudiar la relación con sólo uno de ellos. Podemos asimismo apreciar que las rectas de regresión ajustadas muestran algunas diferencias notorias, y no así las diferencias de distribuciones de cada una de las variables; esto último lo podemos ver en la diagonal principal del gráfico matricial. 


\section{Regresiones por grupo para CP1 y TOT}
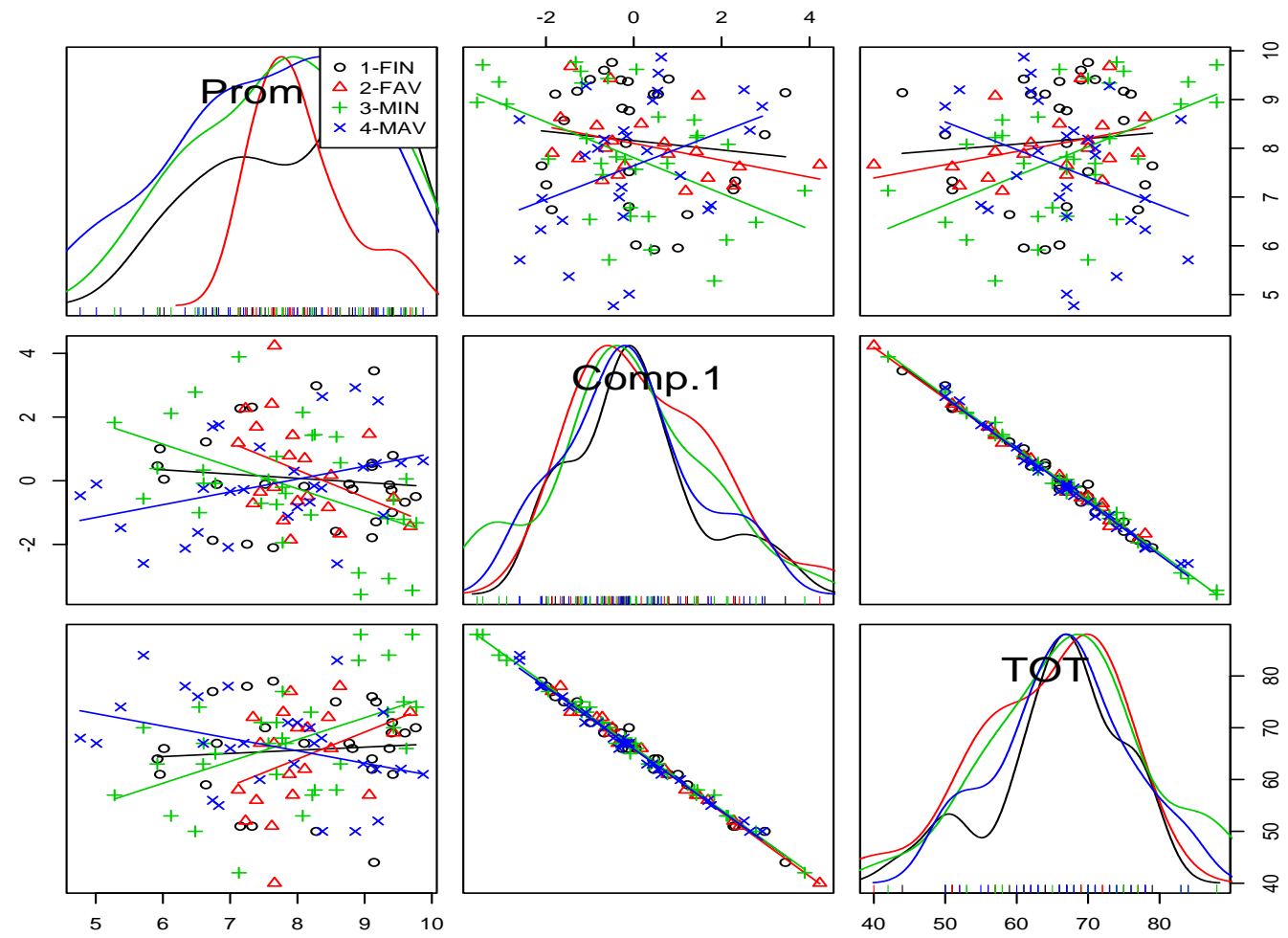

Figura 4. Diagrama matricial que muestra las regresiones ajustadas del promedio con el puntaje total y con el primer componente principal.

La Tabla 1 muestra los resultados del coeficiente de regresión asociado al puntaje total y la significancia correspondiente. Ahí es posible ver que en el grupo de sexo masculino avanzado tiene un patrón que indica que a menor puntaje de HVS el promedio es más alto y viceversa, aunque la significancia es baja (apenas mayor que 0.05). Se destaca el patrón de relación en el grupo de sexo masculino en los semestres iniciales, donde se observa una significancia alta ( $p$ menor que 0.01 ), lo que indica que la relación de puntajes de HVS bajos con promedios bajos, y viceversa, es más nítida. Por otro lado, los grupos de sexo femenino no presentan patrones con nitidez, porque en el caso de las estudiantes de semestres avanzados apenas alcanzan la significancia baja ( $p$ menor que 0.1). Cabe hacer notar que en todos los casos se realizó un análisis de residuos no encontrándose patologías que resolver

Tabla 1. Resultados de los coeficientes de regresión estimados para la pendiente de las rectas por grupo.

\begin{tabular}{|l|l|l|l|l|}
\hline Grupo & Estimador de B1 & Error estándar & Valor de t & Valor p \\
\hline G1=F-INICIAL & 0.01179 & 0.02772 & 0.425 & 0.6743 \\
\hline G2=F-AVANZADO & 0.02720 & 0.01537 & 1.770 & $0.0928^{*}$ \\
\hline G3=M-INICIAL & 0.05996 & 0.01883 & 3.184 & $0.0034^{* *}$ \\
\hline G4=M-AVANZADO & 0.05673 & 0.02810 & 2.019 & $0.0539^{*}$ \\
\hline
\end{tabular}


REVISTA DE LA ESCUELA DE CIENCIAS DE LA EDUCACIÓN, AÑO 16, NRO. 15, VOL. 2, JULIO A DICIEMRE DE 2020. PÁGINAS 34-44. ISSN 2362-3349 (EN LÍNEA). HÁBITOS DE VIDA SALUDABLES Y RENDIMIENTO ESCOLAR EN ESTUDIANTES UNIVERSITARIOS. MARIO MIGUEL OJEDA RAMÍREZ. JOSÉ JUAN MUÑOZ LEÓN. ERNESTO PEDRO MENÉNDEZ ACUÑA

\section{Discusión y conclusiones}

Se ha demostrado que existe una relación entre los HVS y el rendimiento escolar, pero que esta relación está mediada por el sexo y el nivel de avance en los estudios, encontrándose un grupo importante de opiniones contradictorias en los HVS y una relación inversa, la cual podría ser espuria. Esto nos pone en acuerdo con Sánchez-Ojeda y De Luna-Bertos (2015) en el sentido de que a pesar de que los estudiantes conocen la importancia de los HVS, su comportamiento --y nosotros agregamos: a pesar de ser estudiantes de alto rendimiento académico-- es contrario. Podemos concluir que este estudio deja abiertas una serie de interrogantes, pero una aseveración fundamental: es claro que para estudiar la relación de HVS con el rendimiento escolar se necesita considerar otras variables, como las de sexo y nivel de avance en los estudios. En este sentido, y en acuerdo con Díaz Cárdenas et al. (2017), observamos que analizar la relación entre los HVS y el rendimiento escolar exige una visión global del desarrollo académico del estudiante abordando el tema desde una dimensión, al mismo tiempo, personal, ambiental y social. Por otro lado, resulta interesante señalar que en nuestro estudio la actividad y el ejercicio físico (AE) no mostró relación clara como elemento predictor del desempeño escolar; esto nos pone en acuerdo con Taras (2005) y con Yu et al. (2006). No obstante, los resultados que han reportado Capdevila, Bellmunt \& Hernando (2015) así como Shariati y Bakhtiari (2011), justo en el sentido contrario a los hallazgos aquí reportados, nos hacen pensar en extender la discusión en esta línea afinando los instrumentos para recoger datos y ampliando la muestra con estudiantes de la propia unidad académica de economía y estadística. Por último, no hemos encontrado diferencias entre carreras, quizá porque todas las que se incluyeron pertenecen a una misma área de conocimiento; resultaría interesante comparar estos resultados con los que se obtengan en carreras del área de ciencias de la salud. Hay estudios que consideran otros hábitos que están más estrechamente relacionados con los resultados escolares, como hábitos de estudio, o práctica de un deporte, lo cual también plantea otro elemento a considerar en futuros estudios.

\section{Referencias bibliográficas}

Alsabih, M. I., et.al. (2018). The impact of physical activity on health care student academic performance in Riyadh, Saudi Arabia. En: IAIM, 5(2), pp. 30-37.

Capdevila Seder, A.; Bellmunt Villalonga, H. y Hernando Domingo, C. (2015). Estilo de vida y rendimiento académico en adolescentes: comparación entre deportistas y no-deportistas. En: Retos, 27,pp. 28-33.

Cutillas, A. B.; Pérez, F. y Zamora, S. (2013). Calidad de la grasa de la dieta de un colectivo de estudiantes de la Universidad de Murcia. En: II Jornadas de Inicio a la Investigación de Estudiantes de la Facultad de Biología. Universidad de Murcia.

Deliens, T.; Clarys, P., De Bourdeaudhuij, I., y Deforche, B. (2013). Weight, socio-demographics, and health behaviour related correlates of academic performance in first year university students. En: Nutrition journal, 12(1), 162.

Díaz Cárdenas; S., Martínez Redondo, M. \& Zapata Teherán, A. M. (2017). Rendimiento académico y calidad de vida relacionada con la salud en estudiantes de odontología. En: Salud Uninorte. Barranquilla, 33 (2), pp.139-151.

Faught, E. L., et. al. (2017). The combined impact of diet, physical activity, sleep and screen time on academic achievement: a prospective study of elementary school students in Nova Scotia, Canada. En: International Journal of Behavioral Nutrition and Physical Activity, pp.14-29.

Gallardo-Escudero, A., et. al. (2015). La etapa universitaria no favorece el estilo de vida saludable en las estudiantes granadinas. En: Nutrición Hospitalaria, 31(2), pp.975-979.

Gillis, A. J. (1997). The adolescent lifestyle questionnaire: development and psychometric testing. En: Canadian Journal of Nursing Research Archive, 29(1), pp. 29-46.

Guerrero, G., et. al. (2014). Diseño y validación de un cuestionario de hábitos de vida de alimentación y actividad física para escolares de 8-12 años. En: Revista Chilena de Salud Pública, 18(3), pp. 249-256.

Lema, L. F., et. al. (2009). Comportamiento y salud de los jóvenes universitarios: satisfacción con el estilo de vida. En: Pensamiento Psicológico, 5(12):pp.71-88.

Rodríguez, H.; Restrepo, L. F. y Deossa-Restrepo, G. C. (2015). Conocimientos y prácticas sobre alimentación, salud y ejercicio en universitarios de Medellín, Colombia. En: Perspectivas de Nutrición Humana, 17, pp. 36-54.

Sánchez-Ojeda; M. A. y De Luna-Bertos, E. (2015). Hábitos de vida saludable en población universitaria. En: Nutrición Hospitalaria, 31(15), pp. 1910-1919.

Shariati, M. y Bakhtiari, S. (2011). Comparison of personality characteristics athlete and non-athlete student. Islamic Azad University of Ahvaz. En: Social and Behavioral Sciences, 30(0), pp. 2312- 2315.

Taras, H. (2005). Physical activity and student performance at school. Journal of school health, 75(6), pp. 214-218.

Trockel, M. T.; Barnes, M. D. y Egget, D. L. (2000). Health-related variables and academic performance among firstyear college students: Implications for sleep and other behaviors. En: Journal of American college health, 49(3), pp.125-131.

Walker, S.; Sechrist K. y Pender, N. (1987). The health-promoting lifestyle profile: development and psychometric characteristics. Nursing Research, 36(2), pp. 76-81. 
REVISTA DE LA ESCUELA DE CIENCIAS DE LA EDUCACIÓN, AÑO 16, NRO. 15, VOL. 2, JULIO A DICIEMRE DE 2020. PÁGINAS 34-44. ISSN 2362-3349 (EN LÍNEA). HÁBITOS DE VIDA SALUDABLES Y RENDIMIENTO ESCOLAR EN ESTUDIANTES UNIVERSITARIOS. MARIO MIGUEL OJEDA RAMÍREZ. JOSÉ JUAN MUÑOZ

LEÓN. ERNESTO PEDRO MENÉNDEZ ACUÑA

Yu, C. C. W., et. al. (2006). Are physical activity and academic performance compatible? Academic achievement, conduct, physical activity and self-esteem of Hong Kong Chinese primary school children. En: Educational Studies, 32(4), pp. 331-341. 